\title{
Noradrenergic Function and Clinical Outcome in Antidepressant Pharmacotherapy
}

\author{
Helen L. Miller, M.D., R. David Ekstrom, M.A., M.P.H., George A. Mason, Ph.D.,
} R. Bruce Lydiard, M.D., Ph.D., and Robert N. Golden, M.D.

Controversy remains regarding the role of noradrenergic systems in determining clinical response to antidepressant pharmacotherapy. Pineal gland production of melatonin can serve as a physiologic index of noradrenergic function. The aim of this study was to examine the effects of antidepressant treatment on 24-hour urinary excretion of the principle metabolite of melatonin, 6-sulfatoxymelatonin in treatment responders and nonresponders. Twenty-four outpatients meeting DSM-III-R criteria for Major Depression received treatment with either fluvoxamine or imipramine for 6 weeks while participating in a placebocontrolled double-blind clinical trial. Twenty-four hour excretion of 6-sulfatoxymelatonin was measured at baseline and at the conclusion of the treatment trial. Changes in urinary excretion of 6-sulfatoxymelatonin distinguished antidepressant responders from nonresponders, with a significant increase observed in the former group and a significant decrease in the latter. The degree of clinical response was correlated with the change in 6-sulfatoxymelatonin excretion. These results suggest that enhanced noradrenergic function may play an important role in determining clinical response to antidepressant pharmacotherapy.

[Neuropsychopharmacology 24:617-623, 2001] (C) 2001 American College of Neuropsychopharmacology. Published by Elsevier Science Inc.
KEY WORDS: Antidepressants; Melatonin; Norepinephrine; Pineal gland; Depression

The introduction of effective antidepressant pharmacotherapies in the 1950s revolutionized the treatment of mood disorders. However, despite several decades of research, the mechanisms of action of antidepressants remain unclear. Elucidation of these mechanisms is of considerable clinical, as well as scientific, importance. Although the majority of patients respond to treatment,

From the Department of Psychiatry (HLM, RDE, GAM, RNG) University of North Carolina School of Medicine, Chapel Hill, North Carolina, and the Department of Psychiatry (RBL), Medical University of South Carolina, Charleston, South Carolina.

Address correspondence to: Robert N. Golden, M.D., Psychiatry Department, Campus Box \# 7160, University of North Carolina, Chapel Hill, NC 27599-7160. Tel.: 919-966-4738; Fax: 919-966-7659; E-mail: rgolden@css.unc.edu

Received 2 August 2000; revised 28 October 2000; accepted 2 November 2000. a substantial minority do not, and insights regarding the necessary psychobiologic components of treatment response could help predict, and perhaps prevent, some cases of treatment failure.

Early observations focused attention on the role of norepinephrine (NE) in determining antidepressant response. All of the original antidepressants, including the tricyclic antidepressants (TCAs) and monoamine oxidase inhibitors (MAOIs), enhance noradrenergic neurotransmission, either directly or via active metabolites. Based on these observations, early theories of antidepressant action proposed that clinical response is related to increased noradrenergic activity in the brain (Schildkraut 1969). Later studies found that most antidepressants, including those with minimal direct biochemical effects on NE, can influence noradrenergic systems. For example, lithium, electroconvulsive therapy, the dopamine reuptake inhibitor bupropion, and the serotonin (5-HT) reuptake inhibitor zimelidine all decrease "whole-body norepinephrine turnover"; that 
is, the 24-hour excretion of NE and its major metabolites (Linnoila et al. 1983; Rudorfer et al. 1984; Golden et al. 1988). Central NE turnover, as measured by cerebrospinal fluid concentrations of the NE metabolite 3-methoxy-4-hydroxy-phenylglycol (MHPG), is also reduced by a variety of antidepressants, including the 5-HT specific reuptake inhibitor zimelidine (Potter et al. 1985). Based on these observations, it seems that increased noradrenergic function is an integral component of antidepressant treatments.

On the other hand, a wide variety of antidepressants, including tricyclic NE and 5-HT reuptake inhibitors, monoamine oxidase inhibitors, and repeated electroconvulsive shock, down-regulate the density of $\beta$-adrenergic receptor $(\beta A R)$ ligand-binding sites in limbic brain regions, including the cerebral cortex and the hippocampus (Vetulani and Sulser 1975; Banerjee et al. 1977; Sugrue 1983). Furthermore, nearly all antidepressants diminish the ability of $\beta$ ARs to stimulate adenylate cyclase-mediated cAMP production in these regions (Vetulani and Sulser 1975; Charney et al. 1981), and the time course for these receptor changes is consistent with the time course for clinical response to antidepressant treatment. Together, these observations have been cited as support for an alternative hypothesis of antidepressant action, which proposes that $\beta$ AR receptor down-regulation, and the consequent decrease in noradrenergic function, is responsible for antidepressant action (Sulser et al. 1978; Charney et al. 1981). In addition, antidepressants decrease tyrosine hydroxylase and tyrosine hydroxylase mRNA following chronic administration (Nestler et al. 1990), and chronic exposure to either desipramine or electroshock decreases norepinephrine transporter binding (Bauer and Tejani-Butt 1992). These observations lend further support to the hypothesis that antidepressant response is linked to decreased noradrenergic function (Owens 1996, 1997).

There are limits to our ability to resolve these conflicting theories of antidepressant mechanism of action by means of approaches that examine isolated, static components of the dynamic NE neurotransmitter system. The most careful exploration of presynaptic events or postsynaptic responses will not completely clarify the net physiologic effects of antidepressant treatments in humans. Rather, what is needed is a physiologic gauge of over-all noradrenergic function that integrates presynaptic and postsynaptic events, including those events that are "downstream" from receptors, and can be applied to clinical studies (Golden and Potter 1986).

Pineal gland production of melatonin is, in many ways, well suited to serve this purpose. The pineal gland is unique in that, unlike other endocrine organs in the human body, it is regulated primarily by neural (sympathetic) input and does not seem to be affected by circulating hormones to any appreciable degree (Lewy 1984). Stimulation of $\beta$ ARs on the pineal gland triggers a cascade of events (including increased activity of n-acetyltransferase, the rate-limiting step in melatonin production) that lead to the synthesis of melatonin (Axelrod 1974) (see Figure 1). Pharmacologic blockade of BARs suppresses melatonin production (Arendt et al. 1985; Cowen et al. 1983). Stimulation of $\alpha$-adrenergic receptors potentiates the effects of $\beta A R$ stimulation on melatonin synthesis (Klein et al. 1983; Sugden et al. 1984; Sugden et al. 1985). Thus, pineal gland melatonin production can provide a physiologic index of noradrenergic activity. In this regard, Axelrod has referred to the pineal gland as a "biochemical transducer," in recognition of its capacity to convert noradrenergic activity into the production of melatonin (Axelrod 1974).

Pineal gland activity can be assessed by repeated measuring of plasma melatonin concentration over a 24-hour period and calculating the area under the curve for such samples. Alternatively, urinary excretion of the principal metabolite of melatonin can provide a more practical approach for determining net pineal gland activity in psychiatric populations. Melatonin is metabolized in the liver, and approximately $85 \%$ is excreted as the sulfated (6-sulfatoxymelatonin) and glucuronidated conjugates of 6-hydroxymelatonin (Arendt et al. 1985; Markey et al. 1985). There is a robust correlation between nocturnal plasma melatonin concentrations and urinary excretion of 6-sulfatoxymelatonin (6-s-melatonin) (Arendt et al. 1985), and intra-individual excretion rates of urinary melatonin metabolites are consistent over time (Bojkowski et al. 1987). Thus, measurement of 24-hour urinary excretion of 6-s-melatonin provides an index for assessing changes in noradrenergic function, and can thereby serve as a useful tool for examining the net effect of antidepressant treatment on noradrenergic systems.

In an earlier study, we found that diverse antidepressant pharmacotherapies, including the norepinephrine reuptake inhibitor desipramine, the monoamine oxidase inhibitors clorgiline and tranylcypromine, and the dopamine reuptake inhibitor bupropion, enhance 6-hydroxymelatonin output, while diminishing whole body norepinephrine turnover (Golden et al. 1988). However, that study did not examine the relationship between these changes in noradrenergic systems and clinical response. In the current study, we explore the relationship between clinical response and changes in 6-s-melatonin output in depressed patients participating in a controlled trial of a serotonin selective reuptake inhibitor, fluvoxamine, and the tricyclic imipramine.

\section{METHODS}

All subjects gave oral and written informed consent before entry in the study. Patients meeting DSM-III-R criteria for Major Depression were randomly assigned to receive treatment with either fluvoxamine $(n=13)$, imipramine $(n=11)$, or placebo $(n=13)$, under doubleblind conditions. Doses were titrated based on patient 


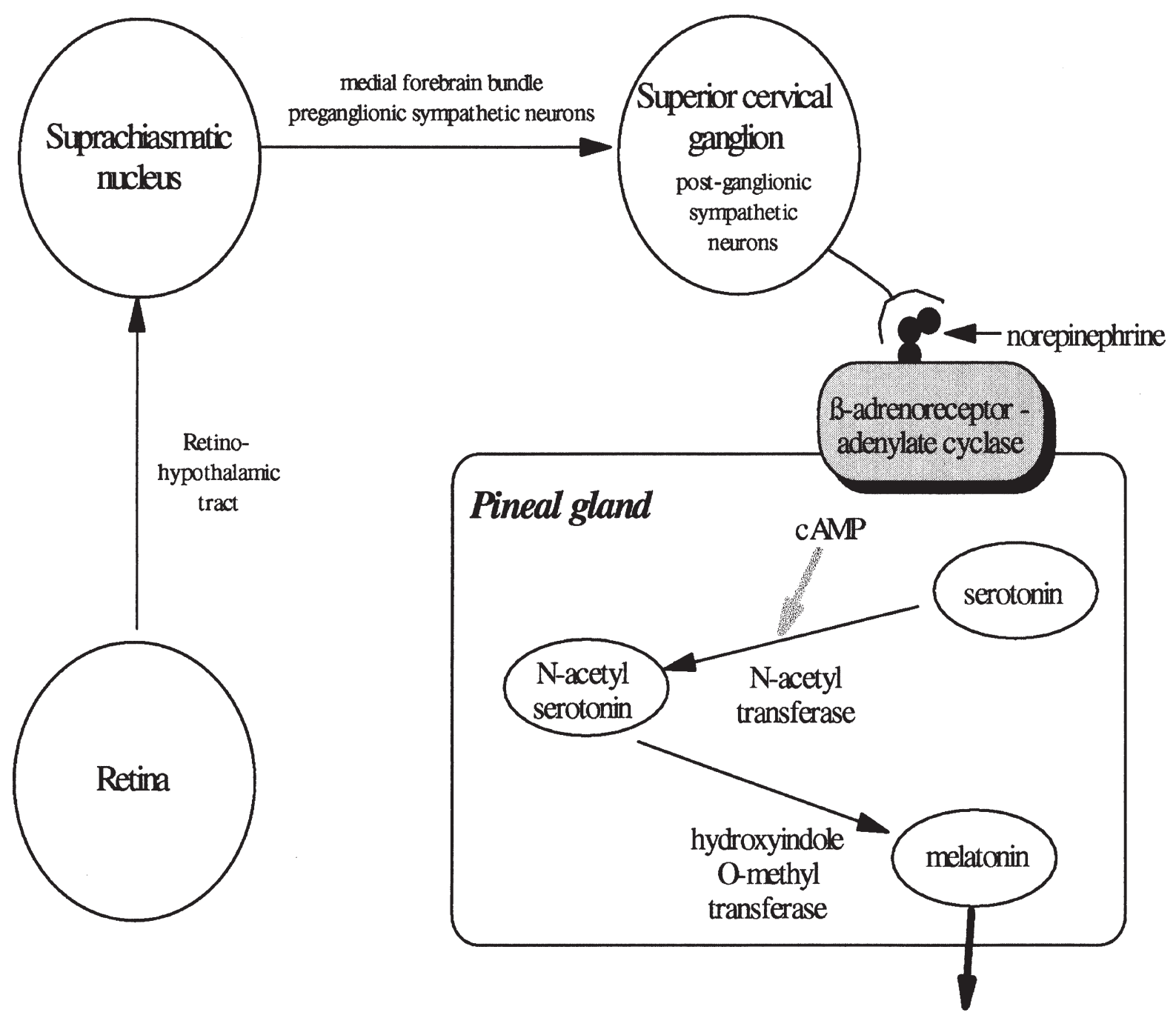

Figure 1. Regulation of pineal gland production of melatonin is under noradrenergic control.

tolerance of side effects. Hamilton depression rating scale (HDRS) scores were obtained by a research clinician unaware of treatment assignment. "Treatment responders" were defined as those patients whose HDRS scores decreased by more than $50 \%$ from baseline and whose final HDRS scores were less than 15 following 6 weeks of treatment; "nonresponders" were those patients who failed to meet these criteria. Recognizing that the psychobiology and mechanism of action of placebo response may be different from that associated with active treatments, we planned a priori to analyze treatment-associated changes in 6-s-melatonin output in those subjects who received active pharmacological treatment. We also planned a secondary analysis of the placebo group.

Twenty-four hour output of 6-s-melatonin was measured in coded urine samples by laboratory personnel who were blind to study condition. A modification of the radioimmunoassay procedure of Arendt et al. (1985) was used, utilizing reagents that were supplied by CID Tech Research Inc. (Mississauga, Ontario, Canada). Using an ${ }^{125}$ I-labeled tracer, the assay has a sensi- tivity of $4 \mathrm{pg} / \mathrm{ml}$ (for urine diluted 1:250) and intraassay and interassay coefficients of variability of 8 and $10 \%$, respectively.

6-S-melatonin change scores (post-treatment minus pretreatment) were calculated for each subject and expressed as a percentage of that subject's pretreatment level. Because of positively skewed distributions of these values, the nonparametric Wilcoxon Signed Rank (within-subject) and Rank Sum (independent groups) tests were used for analyses. Spearman's rank correlation coefficient was used to measure the association between change in 6-s-melatonin output and change in HDRS scores. $P$-values were two-tailed.

\section{RESULTS}

Of the 24 patients receiving an active treatment, 16 met criteria for "responders," and eight were "nonresponders." 6-S-melatonin output increased significantly in the responders, rising from a median baseline level 
of $3.9 \mu \mathrm{g} / 24-\mathrm{h}$ to a post-treatment level of $7.1 \mu \mathrm{g} / 24-\mathrm{h}$ (Wilcoxon Sign Rank, $p=.03$ ). In contrast, "nonresponders" showed a significant decrease in 6-s-melatonin output following treatment (Wilcoxon Sign Rank, $p=$ $.03)$, with median levels dropping from $4.3 \mu \mathrm{g} / 24-\mathrm{h}$ at baseline to $1.2 \mu \mathrm{g} / 24-\mathrm{h}$ following treatment. The difference in treatment-associated changes in 6-s-melatonin output between responders and nonresponders was significant (Wilcoxon Rank Sum, $p=.01$ ). The degree of clinical response was related to the change in 6-s-melatonin excretion. A significant inverse correlation was found between change in HDRS scores and change in 6-s-melatonin output $\left(\mathrm{r}_{\mathrm{S}}=-0.47, p=.02\right.$; see Figure 2$)$.
There was a modest decline in 6-s-melatonin output in depressed patients receiving placebo, which was not statistically significant (Wilcoxon Sign Rank, $p=.38$ ).

\section{DISCUSSION}

These findings provide important new evidence in support of the hypothesis that increased noradrenergic function, as reflected in enhanced 6-s-melatonin output, is linked to clinical response to antidepressant therapy. The observed enhancement in pineal gland activity cannot be attributed to direct pharmacologic effects on NE

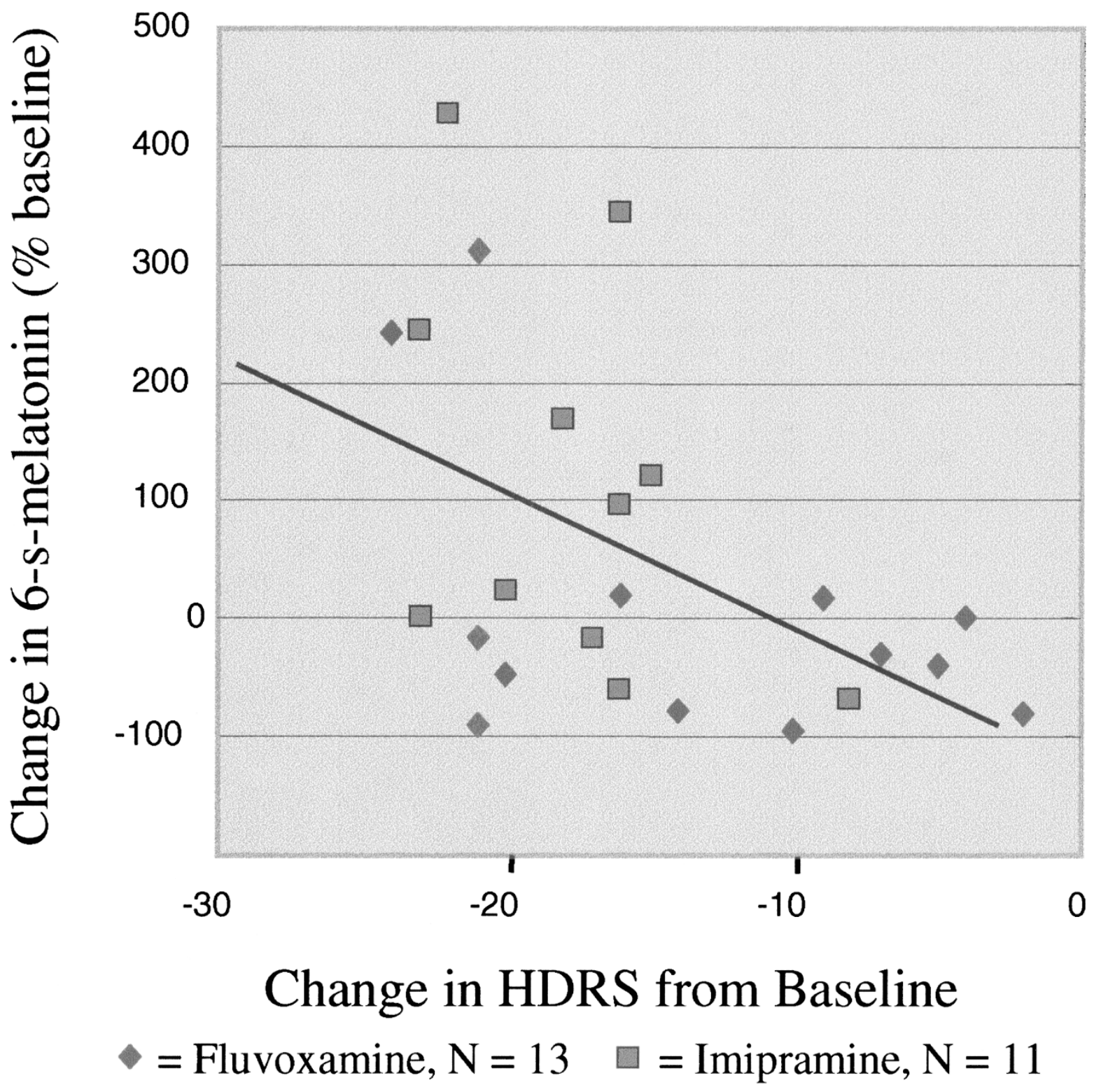

Figure 2. Changes in 6-s-melatonin output show a significant inverse relationship with changes in Hamilton Depression Rating Scale scores following pharmacotherapy. Clinical improvement (i.e., decreases in HDRS scores) is associated with increases in 6-s-melatonin output. 
neurotransmission, because one of the treatment agents, fluvoxamine, is a 5-HT specific reuptake inhibitor with no appreciable direct effects on NE uptake (Tollefson and Rosenbaum 1998). Also, 6-s-melatonin output was enhanced significantly in the subgroup of antidepressant responders, but not in the nonresponders subgroup, suggesting that increased noradrenergic function is an important determinant of clinical response to these antidepressant therapies and not simply a coincidental biochemical effect.

Although, to our knowledge, this is the first study to examine specifically the relationship between clinical response to antidepressant treatment and change in noradrenergic function, as assessed by 6-s-melatonin output, extrapolations from other investigations support our findings. Bearn et al. (1989) also measured 6-s-melatonin excretion in eight depressed patients before and after treatment with the NE reuptake inhibitor desipramine. They observed a significant increase after 1 week, which was sustained after 2 and 3 weeks of treatment. As a group, the patients showed a significant decrease in their HDRS scores, suggesting that depressed patients who respond to antidepressant treatment demonstrate enhanced 6-s-melatonin output. However, no information is given regarding individual clinical responses to desipramine, or how clinical response related to changes in 6-s-melatonin output in their patients. Sack and Lewy (1986) also found that desipramine treatment was associated with an increase in 6-hydroxymelatonin output in four depressed patients, although they did not report the clinical outcome of treatment. Brown et al. (1996) also found a significant increase in 6-s-melatonin output in 21 depressed outpatients following 5 weeks of treatment with desipramine in an unblinded study. Although Hamilton depression scores significantly declined following treatment, there was no statistically significant correlation between the changes in these scores and the changes in 6-s-melatonin output. An earlier study by the same group reported a significant increase in 6-s-melatonin output following 1 week of treatment with desipramine in nine patients, followed by a return toward baseline levels at 6 weeks. It should be noted that only two of the patients would have met our criteria for "responder" at 6 weeks. Also in that study, there was not significant change in 6-s-melatonin in six patients (two of whom would meet our criteria for "responder") following 6 weeks of treatment with adinazolam (Kennedy and Brown 1992).

Several other investigations have examined the effects of antidepressant treatments on plasma melatonin secretion in depressed patients. Jarrett et al. (1988) found that acute, subtherapeutic doses of imipramine (e.g., $50 \mathrm{mg}$ ) did not increase nocturnal melatonin secretion in depressed patients, so that simple exposure to antidepressant is insufficient to enhance pineal activity in depressed subjects. Thompson et al. (1985) reported that plasma melatonin concentrations increased in depressed patients after 3 weeks of desipramine treatment, but not after 1 week. This supports the hypothesis that increased melatonin output is linked to clinical response, which usually does not occur within 1 week, but often after 3 weeks of treatment. Palazidou et al. (1992) also observed increased plasma melatonin concentrations in depressed patients following 3 and 6 weeks of treatment with desipramine. As a group, the eight patients in that study showed significant improvement, but the authors did not compare the changes in melatonin secretion in responders versus nonresponders. Wirz-Justice and Arendt (1980) found no increase in plasma melatonin concentrations in three patients who failed to respond to treatment with the norepinephrine reuptake inhibitor maprotiline. Murphy et al. (1986) reported significant increases in plasma melatonin concentrations in depressed patients following treatment with tranylcypromine or clorgiline (a nonspecific MAO inhibitor and a MAO-A selective inhibitor, respectively), but not following treatment with the MAO-B selective inhibitor deprenyl. Although they do not provide information regarding treatment response, it is interesting to note the tranylcypromine and clorgiline (which increased melatonin concentrations) are effective antidepressants; whereas, deprenyl (which did not increase melatonin concentrations) does not seem to share this property. Frazer et al. (1986) found no significant change in plasma melatonin concentrations in seven patients after 4 weeks treatment with desipramine, but no information is provided regarding clinical outcome. In one other study, plasma melatonin concentrations did not change following treatment with desipramine in seven patients (Thompson et al. 1985). The authors do not describe the treatment outcome, but report relatively low mean plasma concentrations of desipramine (i.e., $70 \pm 9 \mathrm{ng} / \mathrm{ml}$ ), which suggests a lower likelihood of clinical response.

Several caveats should be considered in interpreting our findings. First, because the pineal gland lies outside of the blood brain barrier and receives its innervation from the superior cervical ganglion (Reiter 1981), it serves as a peripheral, rather than a central, marker of noradrenergic function. Also, Den Boer and Westenberg (1990) have speculated that melatonin synthesis may be partially regulated by serotonin, based on their finding that plasma melatonin concentrations in panic disorder patients increased following treatment with the 5-HT uptake inhibitor fluvoxamine. However, as with the observation that treatment with the 5-HT reuptake inhibitor zimelidine reduces cerebrospinal fluid concentrations of the principal NE metabolite 3-methoxy-4-hydroxy-phenylglycol (MHPG) (Potter et al. 1985), this finding seems to suggest that there is considerable "cross-talk" between central NE and 5-HT systems, rather than direct 5-HT regulation of melatonin or MHPG production. 
With the anticipated introduction of second generation norepinephrine specific reuptake inhibitors into clinical in the near future (Gorman and Sullivan 2000), a more precise delineation of the role of noradrenergic function in determining clinical outcome seems timely. Our results suggest that enhanced noradrenergic function, as assessed by pineal gland activity, may play a critical role in determining the success or failure of antidepressant therapy, even for antidepressants that do not have direct effects on norepinephrine uptake or $\mathrm{MAO}$ inhibition.

\section{ACKNOWLEDGMENTS}

This work was supported in part by NIH Grants MH-33127, MH-19111, and RR-00045.

\section{REFERENCES}

Arendt J, Bojkowski C, Franey C, Wright J, Marks Y (1985): Immunoassay of 6-hydroxymelatonin sulfate in human plasma and urine: Abolition of the urinary 24-hour rhythm with atenolol. J Clin Endocrinol Metab 60:11661173

Axelrod J (1974): The pineal gland: A neurochemical transducer. Science 184:1341-1348

Banerjee SP, Kung LS, Riggi SJ, Chanda SK (1977): Development of B-adrenergic receptor subsensitivity by antidepressants. Nature 268:455-456

Bauer ME, Tejani-Butt SM (1992): Effects of repeated administration of desipramine or electroconvulsive shock on norepinephrine uptake sites measured by [3H]-nisoxetine autoradiography. Brain Res 582:208-214

Bearn J, Franey C, Arendt J, Checkley SA (1989): A study of the effects of desipramine treatment alone and in combination with 1-triiodothyronine on 6-sulphatoxymelatonin excretion in depressed patients. $\mathrm{Br} \mathrm{J}$ Psychiat 155:341-347

Bojkowski CJ, Arendt J, Shih MC, Markey SP (1987): Melatonin secretion in humans assessed by measuring its metabolite: 6-sulfatoxymelatonin. Clin Chem 33:13431348

Brown GM, Singer W, Joffe R (1996): Lack of association between thyroid and pineal responses to antidepressant treatment. Depression 4:73-76

Charney DS, Menkes DB, Heninger GR (1981): Receptor sensitivity and the mechanism of action of antidepressant treatment: Implications for the etiology and therapy of depression. Arch Gen Psychiat 38:1160-1180

Cowen PJ, Fraser S, Sammons R, Green AR (1983): Atenolol reduces plasma melatonin concentration in man. Br J Clin Pharmacol 15:579-581

Den Boer JA, Westenberg HGM (1990): Serotonin function in panic disorder: A double-blind placebo-controlled study with fluvoxamine and ritanserin. Psychopharmacology 102:85-94
Frazer AL, Brown R, Kocsis J, Caroff S, Amsterdam J, Winokur A, Sweeney J, Stokes P (1986): Patterns of melatonin rhythms in depression. J Neural Transm Suppl 21:269-290

Golden RN, Markey SP, Risby ED, Cowdry RW, Potter WZ (1988): Antidepressants reduce whole-body norepinephrine turnover while enhancing 6-hydroxymelatonin output. Arch Gen Psychiat 45:150-154

Golden RN, Potter WZ (1986): Neurochemical and neuroendocrine dysregulation in affective disorders. Psychiatr Clin North Am 9:313-327

Golden RN, Rudorfer MV, Sherer M, Linnoila M, Potter WZ (1988): Bupropion in depression: Biochemical effects and clinical response. Arch Gen Psychiat 45:139-143

Gorman JM, Sullivan G (2000): Noradrenergic approaches to antidepressant therapy. J Clin Psychiat 61 (Suppl 1):13-16

Jarrett DB, Miewald JM, Kupfer DJ (1988): Acute changes in sleep-related hormone secretion of depressed patients following oral imipramine. Biol Psychiat 24:541-554

Kennedy SH, Brown GM (1992): Effect of chronic antidepressant treatment with adinazolam and desipramine on melatonin output. Psychiat Res 43:177-185

Klein DC, Sugden D, Weller JL (1983): Postsynaptic $\alpha$-adrenergic receptors potentiate the $\beta$-adrenergic stimulation of pineal serotonin $\mathrm{N}$-acetyltransferase. Proc Natl Acad Sci USA 80:599-603

Lewy A (1984): Human melatonin secretion: I. A marker for adrenergic function. In Post $\mathrm{R}$ and Ballenger $\mathrm{J}$ (eds), Neurobiology of Mood Disorders. Baltimore, Williams \& Wilkins, pp 204-214

Linnoila M, Karoum F, Rosenthal N, Potter WZ (1983): Electroconvulsive therapy and lithium carbonate: Their effects on norepinephrine metabolism in patients with primary major depression. Arch Gen Psychiat 40:677-680

Markey SP, Higa S, Shih M, Danforth DN, Tamarkin L (1985): The correlation between human plasma melatonin levels and urinary 6-hydroxymelatonin excretion. Clin Chim Acta 150:221-225

Murphy DL, Tamarkin L, Sunderland T, Garrick NA, Cohen R (1986): Human plasma melatonin is elevated during treatment with monoamine oxidase inhibitors clorgyline and tranylcypromine but not deprenyl. Psychiat Res 17:119-127

Nestler EJ, McMahon A, Sabban EL, Tallman JF, Duman RS (1990): Chronic antidepressant administration decreases the expression of tyrosine hydroxylase in the rat locus ceruleous. Proc Natl Acad Sci USA 87:7522-7526

Owens MJ (1996, 1997): Molecular and cellular mechanisms of antidepressant drugs. Depress Anxiety 4:153-159

Palazidou E, Papadopoulos A, Ratcliff H, Dawling S, Checkley SA (1992): Noradrenaline uptake inhibition increases melatonin secretion, a measure of noradrenergic neurotransmission, in depressed patients. Psychol Med 22:309-315

Potter WZ, Scheinin M, Golden RN, Rudorfer MV, Cowdry R, Calil H, Ross R, Linnoila M (1985): Selective antidepressants and cerebrospinal fluid: Lack of specificity on norepinephrine and serotonin metabolites. Arch Gen Psychiat 42:1171-1177 
Reiter RJ (1981): The mammalian pineal gland: Structure and function. Am J Anat 162:287-313

Rudorfer MV, Scheinin M, Karoum F, Ross RJ, Potter WZ, Linnoila M (1984): Reduction of norepinephrine turnover by serotonergic drug in man. Biol Psychiat 19:179193

Sack RL, Lewy AJ (1986): Desmethylimipramine treatment increases melatonin production in humans. Biol Psychiat 21:406-410

Schildkraut J (1969): Neuropharmacology and the affective disorders. Second part. N Engl J Med 281:248-255

Sugden D, Namboodiri MAA, Klein DC, Pierce JE, Grady R Jr, Melford (1985): Ovine pineal $\alpha 1$-adrenoceptors: Characterization and evidence for a functional role in the regulation of serum melatonin. Endocrinology 116:1960-1967

Sugden D, Weller JL, Klein DC, Kirk KL, Creveling R (1984): $\alpha$-Adrenergic potentiation of $\beta$-adrenergic stimulation of rat pineal N-acetyltransferase. Biochem Pharmacol 33:3947-3950
Sugrue MF (1983): Chronic antidepressant therapy and associated changes in central monoamine receptor functioning. Pharmacol Ther 21:1-33

Sulser F, Vetulani J, Mobley P (1978): Mode of action of antidepressant drugs. Biochem Pharmacol 27:257-261

Thompson C, Mezey G, Corn TH, Franey C, English J, Arendt J, Checkley SA (1985): The effect of desipramine upon melatonin and cortisol secretion in depressed and normal subjects. Br J Psychiat 147:389-393

Tollefson GD, Rosenbaum JF (1998): Selective serotonin reuptake inhibitors. In Schatzberg AF, Nemeroff $C B$ (eds), The American Psychiatric Press Textbook of Psychopharmacology, 2nd ed., Washington, DC, American Psychiatric Press, Inc., pp 219-237

Vetulani J, Sulser F (1975): Action of various antidepressant treatments reduces reactivity of noradrenergic cyclic AMP-generating system in limbic forebrain. Nature 257:495-496

Wirz-Justice A, Arendt J (1980): Plasma melatonin and antidepressant drugs. Lancet 1:425 